\title{
Aplicaciones de aluminio mantienen el crecimiento de fresa (Fragaria $\times$ ananassa Duch.) suplementada con roca fosfórica en condiciones de cultivo sin suelo
}

\author{
Applications of aluminum sustain growth of strawberry (Fragaria $\times$ ananassa Duch.) supplemented \\ with phosphoric rock in soilless culture
}

\section{Martín Alejandro Tucuch Pérez ${ }^{1}$, Armando Hernández Pérez', Luis Alonso Valdez Aguilar ${ }^{1 \ddagger}$, Gloria Alicia Pérez Arias ${ }^{2}$, Juana Cruz García Santiago ${ }^{1}$ y Daniela Alvarado Carrillo ${ }^{1}$}

\footnotetext{
${ }^{1}$ Departamento de Horticultura, Universidad Autónoma Agraria Antonio Narro. Calzada Antonio Narro 1923. 25315 Saltillo, Coahuila, México.

*Autor responsable (luisalonso.valdez@uaaan.mx)

${ }^{2}$ Facultad de Ciencias Agropecuarias, Universidad Autónoma del Estado de Morelos. Avenida Universidad 1001, 62209 Cuernavaca, Morelos, México.
}

\section{RESUMEN}

Se estima que en los próximos años a nivel mundial habrá carencia de las fuentes de fósforo $(\mathrm{P})$ utilizadas para fabricar los fertilizantes fosforados. Aunque el $P$ puede encontrarse en el suelo, la mayor parte se encuentra en forma no asimilable por las plantas, por lo que para absorberlo éstas exudan ácidos orgánicos (AO) para facilitar su solubilización. Por otro lado, se ha reportado que la presencia de aluminio (Al) se relaciona con un aumento en la exudación de $\mathrm{AO}$ por la raíz. Dado la capacidad solubilizadora de los AO, en el presente estudio se planteó evaluar el efecto de la aplicación de Al sobre la exudación de AO y el uso de $\mathrm{P}$ en forma no soluble (roca fosfórica, RF) en el crecimiento y rendimiento de plantas de fresa. El experimento constó de ocho tratamientos que resultaron de combinar dos cantidades de RF, mezcladas en el sustrato, con aplicaciones de Al. Los tratamientos con RF se irrigaron con una solución que contenía 0.25 meq $\mathrm{L}^{-1} \mathrm{H}_{2} \mathrm{P}_{4}^{-}$. Los resultados mostraron que con aplicaciones de $\mathrm{Al}$ vía riego se obtienen mayores rendimientos, hasta $380 \mathrm{~g}$ planta $^{-1}$, lo cual estuvo correlacionado positivamente con la concentración de ácido cítrico y negativamente con la concentración de ácido málico en el sustrato. Se concluye que el citrato exudado por la raíz debido a la aplicación de $\mathrm{Al}$, favorece el rendimiento de plantas de fresa cuando el $\mathrm{P}$ se encuentra en forma de RF.

Palabras clave: ácidos orgánicos, ácido cítrico, solubilización, cultivos de invernadero.

Cita recomendada:

Tucuch Pérez, M. A., A. Hernández Pérez, L. A. Valdez Aguilar, G. A. Pérez Arias, J. C. García Santiago y D. Alvarado Carrillo. 2017. Aplicaciones de aluminio mantienen el crecimiento de fresa (Fragaria $\times$ ananassa Duch.) suplementada con roca fosfórica en condiciones de cultivo sin suelo. Terra Latinoamericana 35: 193-201.

\section{SUMMARY}

It is estimated that in the near future phosphorus (P) sources for manufacturing fertilizers containing this element will become scarce. Phosphorus can be found in the soil; however, it is not in soluble form for plant uptake. In order to take up this element, plants exude organic acids (OA), which can facilitate the solubilization of $\mathrm{P}$. It has been reported that these acids are exuded into the rhizosphere in the presence of aluminum (Al). Given the solubilizing capacity of OA, the present study was designed to assess the effect of using $\mathrm{Al}$ on exudation of $\mathrm{OA}$, which in turn would improve root uptake of insoluble $\mathrm{P}$ forms, impacting growth and yield of strawberry plants. The experiment consisted of eight treatments that resulted from combining two amounts of phosphoric rock (PR), mixed in the substrate, with applications of Al. The treatments with PR were irrigated with nutrient solutions containing $0.25 \mathrm{meq} \mathrm{L}^{-1} \mathrm{H}_{2} \mathrm{PO}_{4}^{-}$. Application of Al through the irrigation solution produced the highest yields, up to $380 \mathrm{~g}^{\text {plant }}{ }^{-1}$. There was also a positive correlation between yield and citrate concentration and a negative correlation between yield and malate concentration in the substrate. We conclude that increased citrate in the rhizosphere due to the presence of $\mathrm{Al}$ increased solubility of PR, thus increasing yield.

Index words: organic acids, citric acid, solubility, greenhouse crops.

Recibido: octubre de 2016. Aceptado: mayo de 2017. Publicado en Terra Latinoamericana 35: 193-201. 


\section{INTRODUCCIÓN}

El fósforo $(\mathrm{P})$ es un elemento indispensable en el crecimiento y la producción de los cultivos (Vance et al., 2003), al grado de considerarse el segundo elemento más limitante de la producción agrícola a nivel mundial (Holford, 1997), sus funciones no pueden ser sustituidas por otros elementos (White y Hammond, 2008). La principal fuente de $P$ como fertilizante es la roca fosfórica (RF), un recurso no renovable cuyas reservas se estima se agotarán dentro de 100 años (Cordell et al., 2009; Dawson y Hilton, 2011), con lo cual se visualiza un incremento en los precios de los fertilizantes fosforados (Cordell et al., 2011), aunado a problemas de abastecimiento de alimentos para una población mundial estimada en 9 billones de personas para el 2050 (Cordell et al., 2011; Dawson y Hilton, 2011).

En los suelos, el P es uno de los elementos minerales con menor movilidad y disponibilidad para las plantas (Holford, 1997), lo cual limita el crecimiento de los cultivos (Neumman y Römheld, 2007). El P en solución, el cual representa una pequeña fracción del $\mathrm{P}$ total del suelo, es el que se encuentra accesible para las plantas, más del $90 \%$ esta inaccesible y no disponible (Holford, 1997). La baja disponibilidad de P se debe a que es retenido por los minerales del suelo, arcillas o compuestos orgánicos, además de que puede unirse a otros elementos como el hierro (Fe), aluminio (Al) y calcio $(\mathrm{Ca})$, dependiendo del $\mathrm{pH}$ del suelo, formando complejos insolubes (Holford, 1997; Hinsinger, 2001). La baja disponibilidad de $\mathrm{P}$ trae consigo la necesidad de utilizar fertilizantes para aumentar el suministro para los cultivos (Vance et al., 2003), lo que afecta los costos de producción (Kpomblekou-A y Tabatabai, 2003) además de la sobrecarga de los suelos agrícolas y la lixiviación hacia lagos y ríos (Vance et al., 2003). Por lo anterior, es necesario desarrollar estrategias para aumentar la adquisición y la eficiencia en el uso del $\mathrm{P}$ (Vance et al., 2003).

Debido a la baja disponibilidad y movilidad del $\mathrm{P}$, incluso en suelos con niveles adecuados, las plantas pueden llegar a sufrir deficiencias de este elemento (Ryan et al., 2001); sin embargo, algunas especies cuentan con estrategias para asegurar un adecuado suministro (Vance et al., 2003). Una de estas estrategias es la exudación de ácidos orgánicos (AO) ya que, como ha sido reportado por Dinkelaker et al. (1995) y Neumann y Römheld (1999), en condiciones de deficiencia de $\mathrm{P}$ se presenta un incremento en la exudación de estos compuestos (Neumman y Römheld, 2007). No solo las condiciones limitantes de P están relacionadas con un aumento en la síntesis y exudación de $\mathrm{AO}$, sino también la toxcicidad por $\mathrm{Al}$ puede ocasionar respuestas similares (Dinkelaker et al., 1989; Delhaize et al., 1993; Ryan et al., 1995; Jones et al., 1996; Neumann y Römheld, 1999).

Los AO son compuestos de bajo peso molecular que poseen uno o más grupos carboxilo; dependiendo del número de estos grupos presentes en la molécula, los AO llevan una carga negativa variable, por lo que una vez exudados hacia la matriz del suelo forman complejos con cationes o bien pueden desplazar a otros aniones (Jones, 1998). En condiciones de estrés por bajos niveles de $\mathrm{P}$ o presencia de metales tóxicos, los AO, especialmente el ácido cítrico seguido de málico y oxálico, generan un aumento de la disponibilidad de nutrimentos y reducen la concentración de cationes que pueden llegar a ser tóxicos, como el $\mathrm{Al}, \mathrm{Fe}, \mathrm{y} \mathrm{Ca}$, por su capacidad para unirse a estos cationes en la rizósfera (Ryan et al., 2001).

El mecanismo mediante el cual los AO favorecen la liberación de $\mathrm{P}$ de los complejos inorgánicos es mediante un intercambio de ligandos (Raghothama y Karthikeyan, 2005), estos ácidos ocupan los sitios de unión del suelo y forman complejos con elementos como $\mathrm{Al}, \mathrm{Fe}$ y $\mathrm{Ca}$, de modo que el $\mathrm{P}$ no puede unirse a dichos sitios, quedando libre para ser absorbido por las plantas (Ryan et al., 2001). Los AO se han reportado como posibles solubilizadores del $\mathrm{P}$ ya que aumentan la disponibilidad de este elemento cuando se utilizan fuentes poco solubles de $\mathrm{P}$, como lo es la RF (Kpomblekou-A y Tabatabai, 2003; Roy et al., 2016).

Los AO también reducen la toxicidad del Al ya que excluyen su entrada a las raíces; al ser exudados en la zona apical, los ácidos cítrico, oxálico, tartárico y málico forman complejos con el $\mathrm{Al}$, previniendo la entrada de este catión (López-Bucio et al., 2000; Neumman y Römheld, 2007; Jones, 1998; Ryan et al., 2001). La toxicidad por Al ocurre principalmente en suelos ácidos, los cuales abarcan alrededor del 40\% de las tierras cultivables del mundo (Ma et al., 2001), causando una inhibición del crecimiento de las raíces (Delhaize y Ryan, 1995; López-Bucio et al., 2000), incluso aún a concentraciones micromolares (Delhaize y Ryan, 1995), lo que a su vez afecta la adquisión de otros nutrimentos y agua (Ma et al., 2001).

Debido a la baja movilidad del $\mathrm{P}$ y a la predicción 
del agotamiento de los minerales para la producción de fertilizantes fosforados, resulta de interés diseñar estrategias de manejo que permitan aumentar la eficiencia en el uso de este elemento. En sistemas de cultivos sin suelo se emplean sustratos a base de materiales orgánicos o minerales químicamente inertes, por lo que los problemas de fijación/reversión del P son reducidos. Sin embargo, en estos sistemas de producción se utilizan fertilizantes fosforados muy solubles, por lo que la lixiviación del $\mathrm{P}$ es alta comparadas con las perdidas por lixiviación en el suelo (Cartmill et al., 2016); por esta razón es de importancia prestar atención hacia el uso de formas menos solubles de $\mathrm{P}$, como la RF, debido a la alta ineficiencia en sistemas de producción a base de sustratos.

La fresa (Fragaria $\times$ ananassa Duch.) es una especie muy influenciada por la nutrición fosfórica ya que se ha reportado que el crecimiento, número y peso de frutos así como el desarrollo de hojas son favorecidos por altos niveles de este nutrimento (Medeiros et al., 2015). El presente estudio tuvo como objetivo evaluar el uso de RF como fuente de P de baja solubilidad en plantas de fresa cultivadas en sustrato así como aplicaciones de $\mathrm{Al}$ sobre el crecimiento, rendimiento y exudación de AO.

\section{MATERIALES Y MÉTODOS}

\section{Sitio Experimental y Condiciones del Estudio}

El experimento se llevó a cabo en el 2015 en un invernadero del Departamento de Horticultura de la Universidad Autónoma Agraria Antonio Narro, en Saltillo, Coahuila. Durante el experimento, las condiciones ambientales promedio fueron de $19.2{ }^{\circ} \mathrm{C}$ $\left(33.8^{\circ} \mathrm{C}\right.$ máxima y $11.1{ }^{\circ} \mathrm{C}$ mínima) y humedad relativa de $71 \%$ (96\% máxima y $31 \%$ mínima).

Se utilizaron coronas de plantas de fresa cv. San Andreas trasplantadas el 16 de mayo en contenedores de polietileno negro. Los contenedores se llenaron con 6 litros de sustrato a base de turba ácida (Sphagnum peat, Fertilome, Canadá) y perlita grado hortícola (Multiperl Hortícola, Grupo Perlita de la Laguna, México) en una relación 70:30 (v/v). El pH y conductividad eléctrica (CE) del sustrato se ajustó a 6.28 y $0.86 \mathrm{dSm}^{-1}$ agregando $2 \mathrm{~g}$ de $\mathrm{NaHCO}_{3}$ por litro. El exceso de sodio fue eliminado mediante un lavado previo al establecimiento de las plantas.

\section{Diseño de Tratamientos y Análisis Estadístico}

El experimento consistió de ocho tratamientos diseñados a partir de la combinación de dos factores: la dosis de RF (2.5 y $5 \mathrm{~g} \mathrm{~L}^{-1}$ de sustrato) y la forma de aplicación de $\mathrm{Al}$ (mediante el riego o por aspersión foliar). Se empleó RF (00-25-00) con un tamaño de malla 80 mientras que la fuente de Al fue $\mathrm{Al}_{2}\left(\mathrm{SO}_{4}\right)_{3} 18 \mathrm{H}_{2} \mathrm{O}$. Cuando se aplicó en el riego, la concentración de Al utilizada fue de $100 \mu \mathrm{mol} \mathrm{L}^{-1}$, en tanto que por vía foliar fue de $25 \mu \mathrm{mol} \mathrm{L^{-1 }}$ asperjado cada 10 días.

Las plantas se irrigaron con una solución nutritiva que contenía (meq L ${ }^{-1}$ ): $4 \mathrm{NO}_{3}^{-}, 3 \mathrm{SO}_{4}^{2-}, 4.5 \mathrm{Ca}$, $2 \mathrm{~K}$ y $5 \mathrm{Mg}$. Para imponer una condición de baja disponibilidad de $\mathrm{P}$ la solución nutritiva contenía además 0.25 meq $\mathrm{L}^{-1}$ de $\mathrm{H}_{2} \mathrm{PO}_{4}^{-}$. La solución nutritiva fue suministrada mediante un sistema de riego por goteo, ajustando a un $\mathrm{pH}$ de 5.6. El riego fue efectuado de acuerdo a las necesidades hídricas de la planta aplicando el volumen suficiente para obtener un $30 \%$ de fracción de lixiviado.

El diseño experimental utilizado fue completamente al azar con arreglo factorial (dos niveles de RF, cuatro niveles de la forma de aplicación del Al) con 5 repeticiones por cada tratamiento. Los datos obtenidos fueron sometidos a un análisis de varianza (ANOVA) y a una comparación de medias de acuerdo a la prueba de Duncan $(P \leq 0.05)$ utilizando el programa Statistical Analysis System 9 (SAS Institute Inc., USA); además se realizó un análisis de regresión múltiple el cual correlacionó las variables evaluadas con el mismo programa.

\section{Variables Respuesta}

Durante el desarrollo del experimento se podaron las hojas viejas y estolones y se realizó el raleo de flores $\mathrm{y}$ frutos para mantener uniformidad en la cosecha. El peso seco total se determinó a partir de la suma del peso seco de raíces, tallos y hojas (incluyendo hojas podadas). Los órganos vegetales se lavaron con agua desionizada y posteriormente se secaron en un horno a una temperatura de $80^{\circ} \mathrm{C}$ por $72 \mathrm{~h}$ hasta alcanzar un peso constante (DX602, Yamato Scientific Co., Ltd., Japón); posteriormente, estos fueron pesados mediante una balanza analítica (PA1502, Ohaus Corporation, EUA). 
A los cuatro meses de establecido el experimento dio inicio la cosecha de frutos. Para obtener el rendimiento, cada fruto fue pesado individualmente. A los siete meses los frutos cosechados fueron secados en un horno como se mencionó anteriormente para los órganos vegetativos. En frutos con un porcentaje de coloración roja similar se evaluó el contenido de sólidos solubles totales (SST) mediante un refractómetro digital (HI96801, Hanna Instruments Inc., EUA).

\section{Determinación de Ácidos Orgánicos}

La determinación de la concentración de $\mathrm{AO}$ se realizó en una muestra de $30 \mathrm{~g}$ del sustrato tomada a partir de la parte media del cepellón (13 a $17 \mathrm{~cm}$ de profundidad). Esta área de muestreo fue seleccionada debido a que en ella es en donde se presenta la mayor concentración de raíces. A la muestra del sustrato se le agregó $60 \mathrm{ml}$ de agua destilada (relación 1:2) y la mezcla se agitó por $20 \mathrm{~min}$ a $200 \mathrm{rpm}$, y posteriormente se filtró. El filtrado se centrifugó durante $15 \mathrm{~min}$ a $12000 \mathrm{rpm}$ a $4{ }^{\circ} \mathrm{C}$ y fue filtrado nuevamente con un filtro de 0.2 micras previo al análisis en cromatografía líquida de alta resolución (HPLC, Agilent Technologies, Serie 1260 Infinity, Santa Clara, CA, USA) equipado con detector de arreglo de diodos (DAD Agilent 1260 Infinity; modelo G1315D, Santa Clara, CA, USA) y una columna Hypersil GOLD aQ $(250 \mathrm{~mm} \times 4.6 \mathrm{~mm}$, $5 \mu \mathrm{m}$, ThermoScientific, Waltham, MA, USA). E1 estándar de $\mathrm{AO}$ se preparó mediante una solución madre que contenía $10000 \mathrm{mg} \mathrm{L}^{-1}$ de ácido cítrico, málico, oxálico y tartárico respectivamente. La solución madre $\mathrm{y}$ las diluciones se hicieron en agua para HPLC y se almacenaron en oscuridad a una temperatura de $4{ }^{\circ} \mathrm{C}$.

\section{RESULTADOS Y DISCUSIÓN}

Una deficiencia de $\mathrm{P}$ se caracteriza por el desarrollo de una pigmentación roja en las hojas por acumulación de antocianinas, una limitada expansión de la superficie foliar, y un limitado desarrollo de la parte aérea, mientras que el desarrollo de las raíces es menos afectado (Hawskesford et al., 2012). En el presente estudio no se detectaron síntomas de pigmentación ni reducción en el tamaño de las hojas, sin embargo, la producción de biomasa, el rendimiento de fruto (Cuadro 1) y la exudación de AO en la rizósfera (Cuadro 2) fueron afectadas por las dosis de RF y la aplicación de Al. La interacción entre RF y Al afectó significativamente al peso seco (PS) de raíz, de hoja y total (Cuadro 1), además de la concentración de AO en el sustrato (Cuadro 2). Los SST no fueron afectados por ninguno de los factores ni por la interacción (Cuadro 1).

Cuadro 1. Efecto de la forma de aplicación de aluminio vía foliar $\left(25 \mu \mathrm{mol} \mathrm{L}^{-1}\right)$ o con riego $\left(100 \mu \mathrm{mol} \mathrm{L}^{-1}\right)$ en combinación con dos niveles de roca fosfórica sobre el peso seco, rendimiento y sólidos solubles totales (SST) de fruto en plantas de fresa cv. San Andreas.

\begin{tabular}{|c|c|c|c|c|c|c|c|}
\hline \multirow{2}{*}{ Factor } & \multirow{2}{*}{ Niveles } & \multicolumn{4}{|c|}{ Peso seco } & \multirow{2}{*}{ Rendimiento } & \multirow{2}{*}{ SST } \\
\hline & & Tallo & Raíz & Hoja & Total & & \\
\hline & & $\ldots-1$ & $-\cdots$ & - - g pla & $-\ldots$ & $\ldots-\ldots$ & $\%$ \\
\hline \multirow[t]{4}{*}{ Aluminio } & $\operatorname{Sin} \mathrm{Al}$ & 11.4 & 6.38 & $43.3 \mathrm{a}$ & 60.0 & $249 \mathrm{~b}$ & 9.76 \\
\hline & Riego & 10.1 & 6.78 & $43.2 \mathrm{a}$ & 59.5 & $325 \mathrm{a}$ & 9.35 \\
\hline & Foliar & 9.5 & 6.34 & $34.2 \mathrm{~b}$ & 54.5 & $251 \mathrm{~b}$ & 8.83 \\
\hline & Riego $*$ Foliar & 11.0 & 7.12 & $42.9 \mathrm{a}$ & 61.5 & $310 a b$ & 9.06 \\
\hline \multirow[t]{2}{*}{ Roca fosfórica $\left(\mathrm{g} \mathrm{L}^{-1}\right)$} & 2.5 & $11.4 \mathrm{a}$ & 7.06 & $42.5 \mathrm{a}$ & $62.4 \mathrm{a}$ & 299 & 9.19 \\
\hline & 5.0 & $9.6 \mathrm{~b}$ & 6.25 & $39.3 \mathrm{~b}$ & $55.4 \mathrm{~b}$ & 268 & 9.31 \\
\hline \multirow[t]{4}{*}{ ANOVA } & Aluminio & NS & NS & $<0.001$ & NS & 0.047 & NS \\
\hline & Roca fosfórica & 0.003 & NS & 0.027 & 0.002 & NS & NS \\
\hline & Interacción & NS & 0.001 & 0.005 & 0.013 & NS & NS \\
\hline & $\mathrm{CV}$ & 16.46 & 20.57 & 10,13 & 10.97 & 25.46 & 9.97 \\
\hline
\end{tabular}

Medias con diferente letra son diferentes significativamente de acuerdo con la prueba de comparación múltiple de Duncan $(P \leq 0.05)$. NS $=$ no significativo; $\mathrm{ANOVA}=$ análisis de varianza; $\mathrm{CV}=$ coeficiente de variación 
Cuadro 2. Efecto de la forma de aplicación de aluminio (Al) vía foliar $\left(25 \mu \mathrm{mol} \mathrm{L}^{-1}\right)$ o con riego $\left(100 \mu \mathrm{mol} \mathrm{L}^{-1}\right)$ y dos niveles de roca fosfórica en el sustrato sobre la concentración de ácidos orgánicos en la rizósfera de plantas de fresa cv. San Andreas.

\begin{tabular}{|c|c|c|c|c|c|}
\hline Factor & Niveles & Oxálico & Tartárico & Málico & Cítrico \\
\hline & & - - - & $\ldots-m g$ & ato - - & $\cdots$ \\
\hline \multirow[t]{4}{*}{ Aluminio } & $\operatorname{Sin} \mathrm{Al}$ & $135 \mathrm{a}$ & $5.64 \mathrm{bc}$ & $51.6 \mathrm{~b}$ & $0.45 \mathrm{c}$ \\
\hline & Riego & $104 \mathrm{~b}$ & $4.65 \mathrm{c}$ & $39.7 \mathrm{c}$ & $2.27 \mathrm{a}$ \\
\hline & Foliar & $97 \mathrm{~b}$ & $6.99 \mathrm{a}$ & $24.7 \mathrm{~d}$ & $0.15 \mathrm{~d}$ \\
\hline & Riego * Foliar & $75 \mathrm{c}$ & $5.64 \mathrm{ab}$ & $66.2 \mathrm{a}$ & $0.80 \mathrm{~b}$ \\
\hline \multirow[t]{2}{*}{ Roca fosfórica $\left(\mathrm{g} \mathrm{L}^{-1}\right)$} & 2.5 & $109 \mathrm{a}$ & $5.37 \mathrm{~b}$ & $3.8 \mathrm{~b}$ & $1.06 \mathrm{a}$ \\
\hline & 5.0 & $97 \mathrm{~b}$ & $6.24 \mathrm{a}$ & $87.3 \mathrm{a}$ & $0.76 \mathrm{~b}$ \\
\hline \multirow[t]{4}{*}{ ANOVA } & Aluminio & $<0.001$ & 0.005 & $<0.001$ & $<0.001$ \\
\hline & Roca fosfórica & 0.004 & 0.05 & $<0.001$ & $<0.001$ \\
\hline & Interacción & $<0.001$ & $<0.001$ & $<0.001$ & $<0.001$ \\
\hline & $\mathrm{CV}$ & 11.70 & 22.74 & 15.74 & 21.39 \\
\hline
\end{tabular}

Medias con diferente letra son diferentes significativamente de acuerdo con la prueba de comparación múltiple de Duncan $(P \leq 0.05)$. NS $=$ no significativo; ANOVA $=$ análisis de varianza; $\mathrm{CV}=$ coeficiente de variación; $\mathrm{INT}=$ interacción .

\section{Peso Seco}

Cuando las plantas recibieron $5 \mathrm{~g}$ de RF se disminuyó el PS de tallo, hoja y total (Cuadro 1). La interacción entre las dosis de RF y la forma de aplicación de $\mathrm{Al}$ se explica por el hecho de que en las plantas sin Al $\mathrm{y}$ en aquellas que recibieron el Al vía foliar, el PS de raíz (Figura 1A), hoja (Figura 1B) y total (Figura 1C) disminuyeron al elevarse la dosis de RF a $5 \mathrm{~g} \mathrm{~L}^{-1}$. En contraste, en plantas que recibieron el $\mathrm{Al}$ vía riego, el PS de estos órganos se elevó al incrementarse la dosis de RF aplicada al sustrato.

En las plantas que recibieron el Al vía riego + foliar se detectó una disminución en el PS de hoja (Figura 1B) y total (Figura 1C) al elevar la dosis de RF. La disminución del PS en plantas con dosis alta de RF pudo deberse a una intoxicación debido a un exceso del P por la cantidad de RF utilizada, lo cual coincide con lo reportado por Choi y Lee (2012) ya que los autores señalan que existe una reducción del PS de la parte aérea de plantas de fresa al aumentar los niveles de P.

La aplicación de Al no aumentó el PS de las plantas, por el contrario, cuando el Al se aplicó vía foliar o bien tanto al riego + foliar, se detectó una reducción en la biomasa. Lo anterior puede deberse a una toxicidad causada por el Al cuando este se aplicó directamente sobre las hojas, ya que estas no cuentan con mecanismos para excluir su absorción. Similares resultados se han reportado en lechuga (Lawson et al., 2015) y rábano (Strzelecki et al., 2010), en los cuales se compararon aplicaciones foliares y al suelo para aumentar los niveles de yodo; asimismo, el aumento en la cantidad de yodo se correlacionó con una disminución de la biomasa en rábano, pimiento y apio (Strzelecki et al., 2010).

El principal síntoma de una toxicidad por $\mathrm{Al}$ es una inhibición del crecimiento radicular (Delhaize y Ryan, 1995; Ma, 2000; Matsumoto, 2000; López-Bucio et al., 2000). Un exceso de $\mathrm{Al}$ en las plantas puede afectar la división celular, al protoplasma y las paredes celulares, así como ocasionar la precipitación de los ácidos nucleicos (Foy et al., 1978), afectándose de esta manera el crecimiento y acumulación de biomasa.

En el presente estudio, a diferencia de lo observado cuando se aplicó vía foliar, el Al aplicado a través del riego estuvo asociado con una ligera recuperación en el PS cuando se emplearon dosis altas de RF (Figura 1). Lo anterior puede deberse a que el P de la RF formó complejos insolubles con el Al (López-Bucio et al., 2000; Vance et al., 2003; Neumman y Römheld, 2007), evitando así que este sea absorbido por las raíces aunque se encuentre en grandes cantidades, como lo reportan Holford (1997), Raghothama (1999) y López Bucio et al. (2000). 


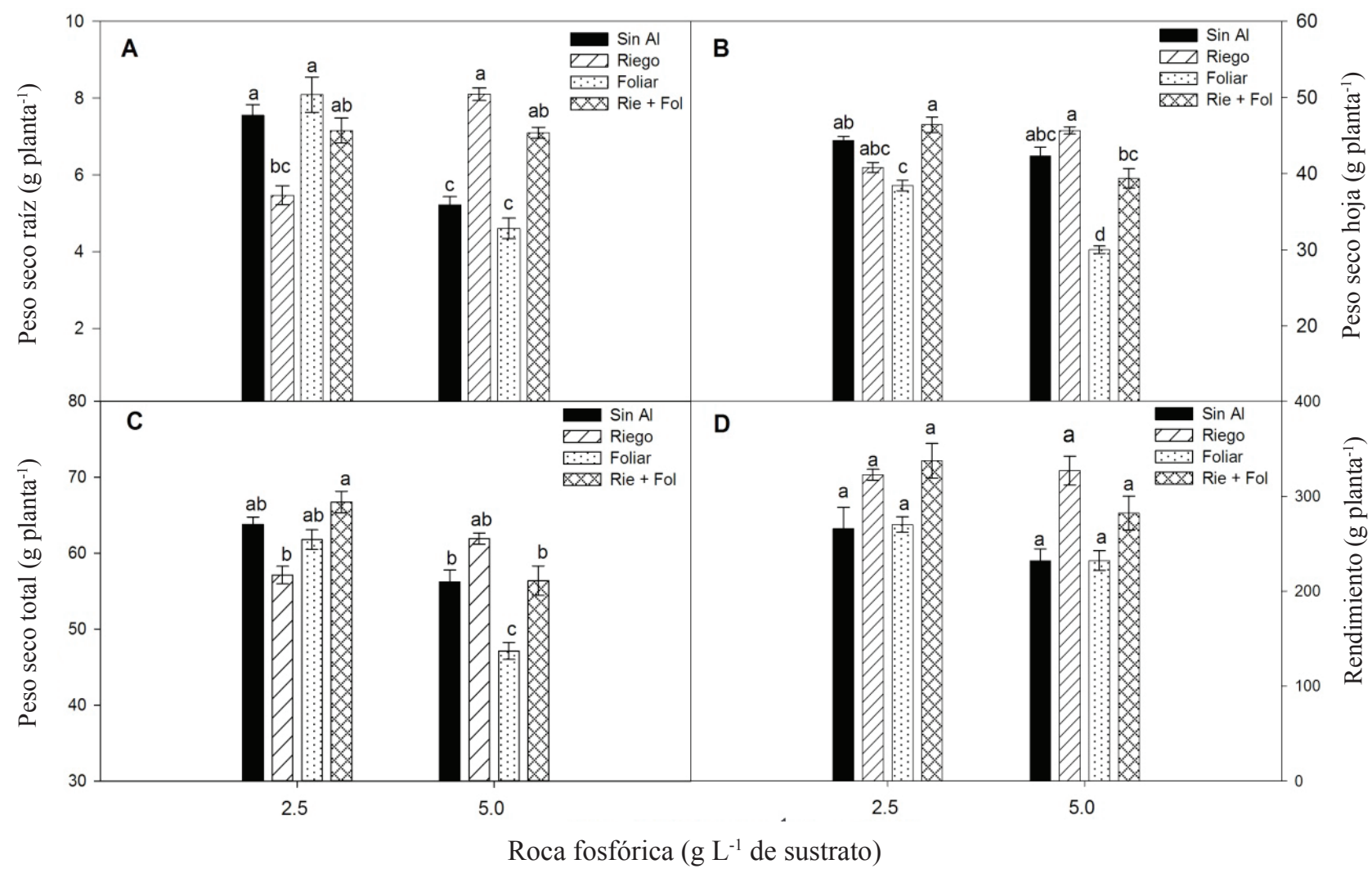

Figura 1. Efecto de la interacción entre la dosis de aplicación de roca fosfórica y las aplicaciones de aluminio (Al) sobre el peso seco de órganos y rendimiento en plantas de fresa cv. San Andreas. Las barras indican el error estándar de la media. Las letras son las categorías obtenidas de la comparación de media con Duncan $(P \leq 0.05)$.

\section{Rendimiento de Fruto}

El rendimiento no fue afectado por la dosis de RF mezclada en el sustrato, sin embargo, la aplicación de Al sí impactó significativamente esta variable, obteniéndose el mayor rendimiento cuando el $\mathrm{Al}$ se aplicó vía riego (31\%) o bien cuando se aplicó vía riego + foliar (24\%) (Cuadro 1, Figura 1D).

Estos resultados sugieren que dosis bajas de $\mathrm{Al}$ permiten a las plantas de fresa un mejor crecimiento y producción de fruto aún y cuando el $\mathrm{P}$ se suministre en forma de RF, siempre que el Al sea aplicado a la zona de las raíces. Lo anterior coincide con lo señalado por Zapata y Roy (2007) quienes señalan que la RF puede emplearse como una fuente de $\mathrm{P}$ de manera directa. Los efectos benéficos de dosis bajas de $\mathrm{Al}$ en el crecimiento de las plantas (Foy et al., 1978) se ha atribuido a la activación de algunas enzimas y mediante el control de algunas propiedades físicas de la membrana plasmática (Kabata-Pendias, 2010).

\section{Ácidos Orgánicos}

La concentración de $\mathrm{AO}$ en el sustrato fue afectada por la interacción entre la forma de aplicación de Al y la dosis de RF (Cuadro 2). La interacción entre los factores sobre la concentración del ácido oxálico (Figura 2A) se debió a que este disminuyó cuando se elevó la dosis de RF en los tratamientos que no recibieron $\mathrm{Al}$ o lo recibieron en el riego, mientras que en los tratamientos con Al aplicado vía foliar o bien al riego + foliar, el contenido del ácido aumentó.

En el caso del ácido tartárico (Figura 2B), la interacción se debió a que las plantas que no recibieron Al y aquellas que lo recibieron vía riego + foliar mostraron una mayor concentración conforme se aumentó la cantidad de RF aplicada en el sustrato. En plantas que recibieron el Al vía foliar, el ácido tartárico disminuyó al reducir la dosis de RF, mientras que en el tratamiento con Al vía el riego no hubo un efecto de la RF. 


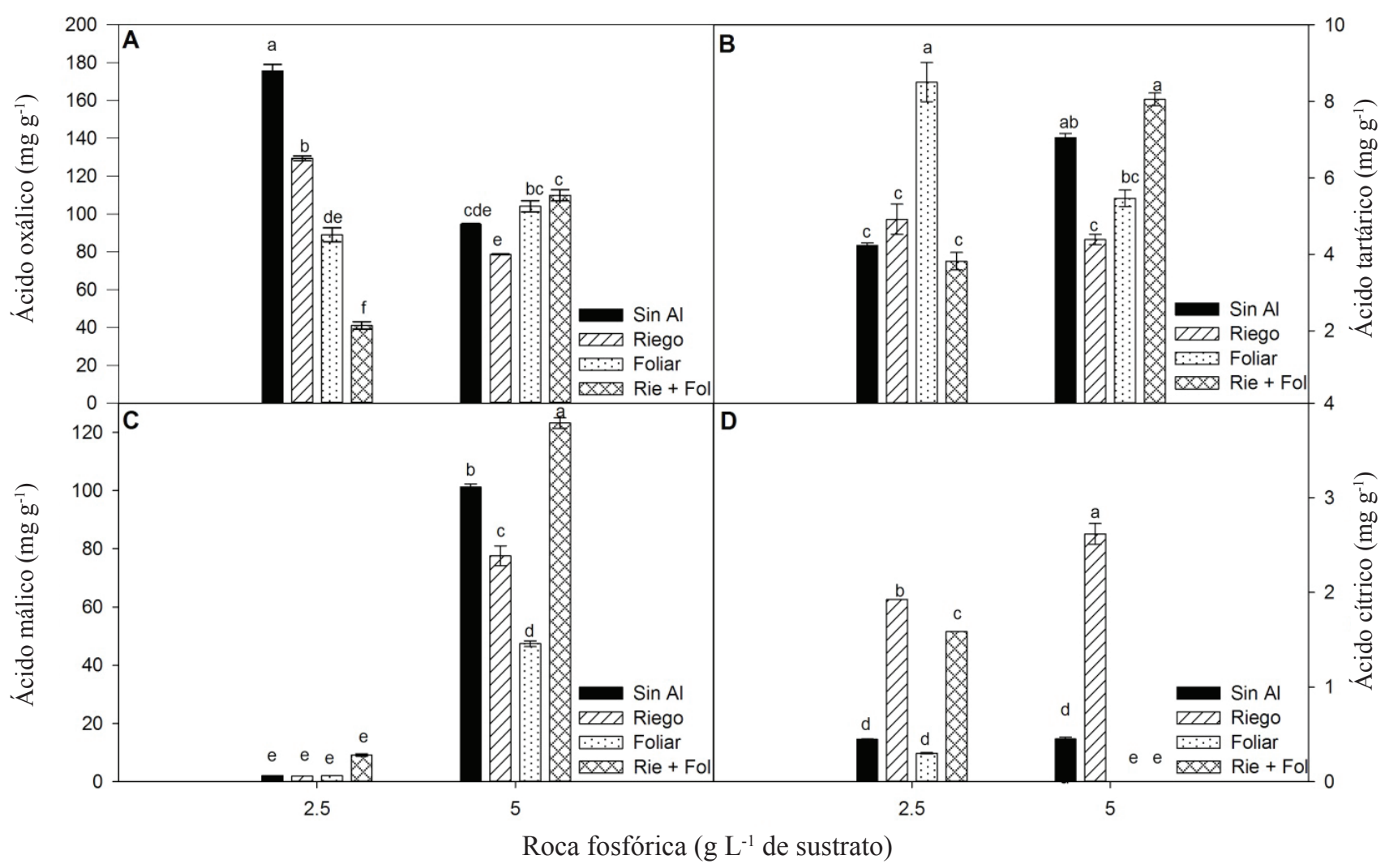

Figura 2. Efecto de la interacción entre la dosis de roca fosfórica y las aplicaciones de aluminio (Al) sobre el contenido de ácido oxálico (A), tartárico (B), málico (C) y cítrico (D) en plantas de fresa cv. San Andreas. Las barras indican el error estándar de la media. Las letras son las categorías obtenidas de la comparación de media con Duncan $(P \leq 0.05)$. NS = ácido no significativo.

El efecto de la interacción sobre el ácido málico (Figura 2C) se debió a que con $2.5 \mathrm{~g}$ de RF todos los tratamientos tuvieron el mismo contenido de ácido en el sustrato, sin embargo, al aumentar la dosis de RF aplicada la concentración de ácido málico aumentó en todos los tratamientos. La interacción para el ácido cítrico se explica porque en las plantas con Al aplicado vía riego existió un aumento en el contenido de este ácido conforme aumenta la dosis de RF, en contraste, en los tratamientos que recibieron Al vía foliar y riego + foliar el contenido del ácido disminuyó (Figura 2D).

El efecto positivo de las aplicaciones de $\mathrm{Al}$ en la exudación de $\mathrm{AO}$ se reflejó en el rendimiento de fruto ya que este se correlacionó con un incremento en la exudación de ácido cítrico (Cuadro 3), lo que sugiere que el Al aplicado al riego indujo la exudación de ácido cítrico, el cual pudo solubilizar la RF presente en el sustrato, favoreciendo la absorción de $\mathrm{P}$ y el aumento en el rendimiento.

El signo negativo en el coeficiente estimado en los modelos de regresión sugiere que en el caso de la fresa, el rendimiento de fruto así como el PS de hojas disminuyen al aumentar la concentración de ácido málico (Cuadro 3), lo cual puede deberse al mecanismo conocido como pH-stat. De acuerdo a Marschner (1995), cuando en la planta existe una presencia

Cuadro 3. Modelos de regresión con significancia entre variable de respuesta y el contenido de ácidos orgánicos en el sustrato.

\begin{tabular}{llrr}
\hline Variable & \multicolumn{1}{c}{ Modelo } & $\mathrm{R}^{2}$ & $\mathrm{Pr}<\mathrm{F}$ \\
\hline Rendimiento & $=252.523-0.344(\mathrm{P}<0.021)[$ málico] $+42.536(\mathrm{P}<0.021)[$ cítrico] & 0.929 & $<0.005$ \\
Peso seco hoja & $=41.535-0.0567(\mathrm{P}<0.0255)[$ málico] & 0.665 & $<0.025$ \\
\hline
\end{tabular}

Los superíndices $\left(\mathrm{p}^{\mathrm{p}<0.000}\right)$ indican el nivel de significancia para el coeficiente respectivo. 
excesiva de aniones se presenta un incremento en el pH del citosol, en este caso, se induce la síntesis de AO con carga negativa, principalmente el malato, el cual estabiliza el pH mediante la compensación de cargas, actuando como un contraión. Por lo tanto, es posible que el Al haya inducido una disminución en la producción de malato, por lo que una disminución en el Al favorece el aumento del rendimiento (junto con el aumento en el citrato). El hecho de que los ácidos oxálico y tartárico no hayan mostrado correlación con alguna de las variables evaluadas puede ser debido a su menor número de grupos carboxilo, por lo que tienen una menor capacidad de actuar como ligandos (Ryan, 2001) y por lo tanto no repercutieron sobre las variables de respuesta.

Ma (2000) y Ma et al. (2001) señalan que en presencia de $\mathrm{Al}$, las plantas secretan los ácidos málico, oxálico y cítrico para proteger a las raíces. El ácido secretado puede variar entre especies, por ejemplo, el ácido málico es secretado por plantas de trigo (Delhaize et al., 1993), el cítrico por frijol (Miyasaka et al., 1991) y maíz (Pellet et al., 1995) y el oxálico por trigo (Zheng et al., 1998b). Es posible también que más de dos tipos de AO puedan ser secretados por una misma planta (Ma, 2000) como ocurre en canola, avena, rábano (Zheng et al., 1998a) y triticale (Ma et al., 2000), las cuales secretan tanto ácido málico como ácido cítrico.

Los AO tienen la capacidad de liberar el $\mathrm{P}$ de los complejos que evitan la absorción a través de un intercambio de ligandos y la disolución y ocupación de los sitios a los cuales el P puede unirse (Raghothama y Karthikeyan, 2005); el ácido cítrico y oxálico son los mas eficientes para solubilizar el $\mathrm{P}$ unido al Fe, Al y Ca (Jones, 1998). Ryan et al. (2001) señalan que los ácidos tricarboxílicos (citrato) tienen una fuerza de quelación superior a los ácidos dicarboxílicos (málico, oxálico) debido al mayor número de grupos carboxilo y la disposición de otras facciones carboxilo e hidroxilo, lo que le confiere estabilidad a los ligandos (BarYosef, 1991; Bolan et al., 1994). En un experimento en el cual se aplicaron AO a diferentes tipos de RF, Kpomblekou-A y Tabatabai (2003) encontraron que los ácidos tricarboxílicos tienen mayor capacidad para solubilizar el $\mathrm{P}$ de la RF, sin embargo, en maíz encontraron que aunque la adición de $\mathrm{AO}$ aumentó la materia seca, no se encontró consistencia sobre cual ácido es mejor, debido a que los $\mathrm{AO}$ se descomponen en pocas horas y su efectividad se ve influenciada por diversos factores.

\section{CONCLUSIONES}

La roca fosfórica $(\mathrm{RF})$ puede emplearse como una fuente de fósforo $(\mathrm{P})$ para producción de fresa en sistemas de cultivo sin suelo, lo que permitiría aumentar la eficiencia en el uso del $\mathrm{P}$ al ser un material poco soluble. Para elevar la solubilidad del $\mathrm{P}$ contenido en la RF, y la disponibilidad del mismo, deben realizarse aplicaciones de aluminio (Al) a dosis de $2.5 \mathrm{~g} \mathrm{~L}^{-1}$ de sustrato vía el riego, lo cual induce la exudación de ácido cítrico, mismo que está relacionado positivamente con el rendimiento de fruto.

\section{LITERATURA CITADA}

Bar-Yosef, B. 1991. Root excretions and their environmental effects: Influence on availability of phosphorus. pp. 529-557. In: Y. Waisel, A. Eshel, and U. Kafkafi (eds.). Plant roots: The hidden half. Marcel Dekker. New York, NY, USA.

Bolan, N. S., R. Naidu, S. Mahimairaja, and S. Baskaran, S. 1994. Influence of low-molecular-weight organic acids on the solubilization of phosphates. Biol. Fertil. Soils 18: 311-319.

Cartmill, A. D., D. L. Cartmill, D. L. Ballweg, and L. A. ValdezAguilar. 2016. Optimum phosphorus concentration for growth of Catharanthus roseus (L.) G. Don 'Pacifica White' in a subirrigation and top watering system. Commun. Soil Sci. Plant Anal. 47: 52-64.

Choi, J. M. and C. W. Lee. 2012. Influence of elevated phosphorus levels in nutrient solution on micronutrient uptake and deficiency symptom development in strawberry cultured with fertigation system. J. Plant Nutr. 35: 1349-1358.

Cordell, D., A. Rosemarin, J. J. Schröder, and A. L. Smit. 2011. Towards global phosphorus security: A systems framework for phosphorus recovery and reuse options. Chemosphere 84 : 747-758.

Cordell, D., J. O. Drangert, and S. White. 2009. The story of phosphorus: Global food security and food for thought. Global Environ. Change 19: 292-305.

Dawson, C. J. and J. Hilton. 2011. Fertiliser availability in a resource-limited world: Production and recycling of nitrogen and phosphorus. Food Pol. 36: S14-S22.

Delhaize, E. and P. R. Ryan. 1995. Aluminum toxicity and tolerance in plants. Plant Physiol. 107: 315-321

Delhaize, E., P. R. Ryan, and P. J. Randall. 1993. Aluminum tolerance in wheat (Triticuma estivum L.) (II. Aluminumstimulated excretion of malic acid from root apices). Plant Physiol. 103: 695-702.

Dinkelaker, B., C. Hengeler, and H. Marschner. 1995. Distribution and function of proteoid roots and other root clusters. Bot. Acta 108: 183-200.

Dinkelaker, B., V. Römheld, and H. Marschner. 1989. Citric acid excretion and precipitation of calcium citrate in the rhizosphere of white lupin (Lupinus albus L.). Plant Cell Environ. 12: 285-292.

Foy, C. D., R. L. Chaney, and M. C. White. 1978. The physiology of metal toxicity in plants. Annu. Rev. Plant Physiol. 29: 511-566. 
Hawskesford, M., W. Horst, T. Kichey, H. Lambers, J. Schjoerring, I. Skumsager Moller, and P. White. 2012. Functions of macronutrients. pp. 135-189. In: P. Marschner (ed.). Marschner's mineral nutrition of higher plants. Academic Press. San Diego, CA, USA.

Hinsinger, P. 2001. Bioavailability of soil inorganic P in the rhizosphere as affected by root-induced chemical changes: A review. Plant Soil 237: 173-195.

Holford, I. C. R. 1997. Soil phosphorus: Its measurement, and its uptake by plants. Aust. J. Soil Res. 35: 227-239.

Jones, D. L. 1998. Organic acids in the rhizosphere-a critical review. Plant Soil 205: 25-44.

Jones, D. L., P. R. Darah, and L. V. Kochian. 1996. Critical evaluation of organic acid mediated iron dissolution in the rhizosphere and its potential role in root iron uptake. Plant Soil 180: 57-66.

Kabata-Pendias, A. 2010. Trace elements in soils and plants. CRC Press. Boca Raton, FL, USA.

Kpomblekou-A, K. and M. A. Tabatabai. 2003. Effect of lowmolecular weight organic acids on phosphorus release and phytoavailabilty of phosphorus in phosphate rocks added to soils. Agric. Ecosyst. Environ. 100: 275-284.

Lawson, P. G., D. Daum, R. Czauderna, H. Meuser, and J. W. Härtling. 2015. Soil versus foliar iodine fertilization as a biofortification strategy for field-grown vegetables. Front. Plant Sci. 6: 450.

López-Bucio, J., M. F. Nieto-Jacobo, V. Ramírez-Rodríguez, and L. Herrera-Estrella. 2000. Organic acid metabolism in plants: From adaptive physiology to transgenic varieties for cultivation in extreme soils. Plant Sci. 160: 1-13.

Ma, J. F. 2000. Role of organic acids in detoxification of aluminum in higher plants. Plant Cell Physiol. 41: 383-390.

Ma, J. F., P. R. Ryan, and E. Delhaize. 2001. Aluminum tolerance in plants and the complexing role of organic acids. Trends Plant Sci. 6: 273-278.

Ma, J. F., S. Taketa, and Z. M. Yang. 2000. Aluminum tolerance genes on the short arm of chromosome 3R are linked to organic acid release in triticale. Plant Physiol. 122: 687-694.

Marschner, H. 1995. Mineral nutrition of higher plants. Academic Press. San Diego, CA, USA.

Matsumoto, H. 2000. Cell biology of aluminum toxicity and tolerance in higher plants. Int. Rev. Cytol. 200: 1-46.

Medeiros, R. F., W. E. Pereira, R. M. Rodrigues, R. Nascimento, J. F. Suassuna, and T. A. G. Dantas. 2015. Growth and yield of strawberry plants fertilized with nitrogen and phosphorus. Rev. Bras. Eng. Agríc. Ambient. 19: 865-870.
Miyasaka, S. C., J. G. Buta, R. K. Howell, and C. D. Foy. 1991. Mechanism of aluminum tolerance in snapbeans: Root exudation of citric acid. Plant Physiol. 96: 737-743.

Neumann, G. and V. Römheld. 1999. Root excretion of carboxylic acids and protons in phosphorus-deficient plants. Plant Soil 211: 121-130.

Neumman, G. and V. Römheld. 2007. The release of root exudates as affected by plant physiological status. pp. 23-72. In: R. Pinton, Z. Varanini, and P. Nannipieri (eds.). The Rhizosphere: Biochemistry and organic substances at the soil-plant interface. CRC Press. Boca Raton, FL, USA.

Pellet, D. M., D. L. Grunes, and L. V. Kochian. 1995. Organic acid exudation as an aluminum-tolerance mechanism in maize (Zea mays L.). Planta 196: 788-795.

Raghothama, K. G. 1999. Phosphate acquisition. Annu. Rev. Plant Physiol. Plant Mol. Biol. 50: 665-693.

Raghothama, K. G. and A. S. Karthikeyan. 2005. Phosphate acquisition. Plant Soil 274: 37-49.

Roy, T., D. R. Biswas, S. C. Datta, and A. Sarkar. 2016. Phosphorus release from rock phosphate as influenced by organic acid loaded nanoclay polymer composites in an alfisol. Proc. Natl. Acad. Sci. India Sect. B: Biol. Sci. DOI 10.1007/s40011-0160739-6.

Ryan, P. R., E. Delhaize, and D. L. Jones. 2001. Function and mechanism of organic anion exudation from plant roots. Annu. Rev. Plant Physiol. Plant Mol. Biol. 52: 527-560.

Ryan, P. R., E. Delhaize, and P. J. Randall. 1995. Malate efflux from root apices and tolerance to aluminium are highly correlated in wheat. Funct. Plant Biol. 22: 531-536.

Strzelecki, P., S. Smoleń, S. Rożek, and W. Sady. 2010. Effect of differentiated fertilization and foliar application of iodine on yielding and antioxidant properties in radish (Raphanus sativus L.) plants. Ecol. Chem. Eng. 17: 1189-1196.

Vance, C. P., C. Uhde-Stone, and D. L. Allan. 2003. Phosphorus acquisition and use: Critical adaptations by plants for securing a nonrenewable resource. New Phytol. 157: 423-447.

White, P. J. and J. P. Hammond. 2008. The ecophysiology of plantphosphorus interactions. Springer. Netherlands.

Zapata, F. y R. N. Roy. 2007. Utilización de las rocas fosfóricas para una agricultura sostenible. FAO. Roma, Italia.

Zheng, S. J., J. F. Ma, and H. Matsumoto. 1998a. Continuous secretion of organic acids is related to aluminium resistance during relatively long-term exposure to aluminium stress. Physiol. Plant. 103: 209-214.

Zheng, S. J., J. F. Ma, and H. Matsumoto. 1998b. High aluminum resistance in buckwheat I. Al-induced specific secretion of oxalic acid from root tips. Plant Physiol. 117: 745-751. 